\title{
FOUNDATIONS OF INFANTS' SOCIAL GROUP EVALUATIONS
}

by

Anthea Colleen Pun

\section{A THESIS SUBMITTED IN PARTIAL FULFILLMENT OF THE REQUIREMENTS FOR THE DEGREE OF \\ MASTER OF ARTS \\ in}

The Faculty of Graduate and Postdoctoral Studies

(Psychology)

THE UNIVERSITY OF BRITISH COLUMBIA

(Vancouver)

August 2015

(C) Anthea Colleen Pun, 2015 


\begin{abstract}
Previous research has suggested that infants exhibit a preference for familiar over unfamiliar social groups (e.g., preferring individuals from their own language group over individuals from a foreign language group). However, it is not clear whether such intergroup preferences are driven by positivity toward the familiar group and/or negativity toward the unfamiliar group. Using a novel habituation paradigm modeled conceptually after the Implicit Association Test, experiments 1-3 demonstrated that infants around 1-year of age positively evaluate the familiar language group, but do not negatively evaluate an unfamiliar language group. Experiments 4-5 addressed alternative interpretations of this core finding. Experiment 6 conceptually replicated Experiments 13, demonstrating that infants also expect members of the familiar language group (but not members of an unfamiliar group) to engage in prosocial behaviours. Together these data suggest that children's early social group behaviours (e.g., toy choice, preferential looking) may be shaped by positive evaluations of familiar group(s), rather than negative evaluations of unfamiliar groups.
\end{abstract}




\section{Preface}

Experiments 1-6 included in this dissertation contains original, unpublished, independent work by the author, A.C. Pun.

The research presented here was approved by the University of British Columbia's

Behavioral Research Ethics Board (Project Title: “The Development of Social

Cognition”, H10-00147). 


\section{TABLE OF CONTENTS}

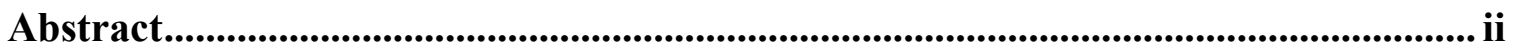

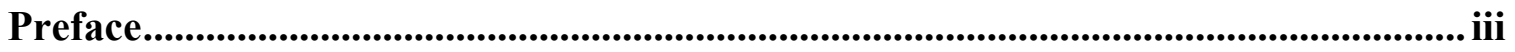

Table of Contents .................................................................................................................... iv

List of Figures................................................................................................................ $\mathrm{v}$

Acknowledgements .......................................................................................................... vi

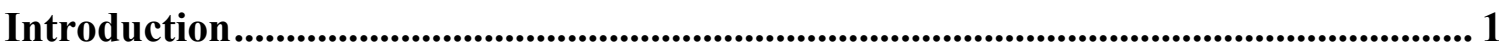

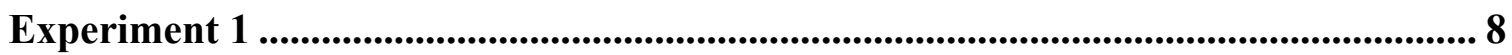

Methods............................................................................................................................... 8

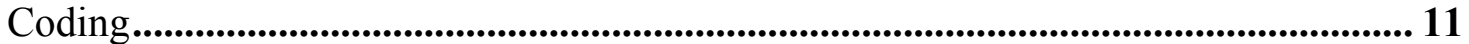

Results and Discussion ............................................................................................. 11

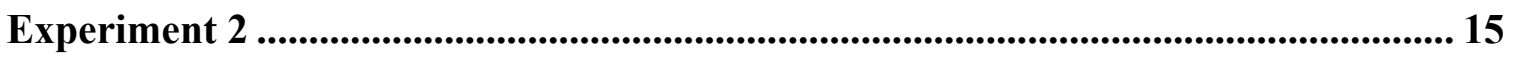



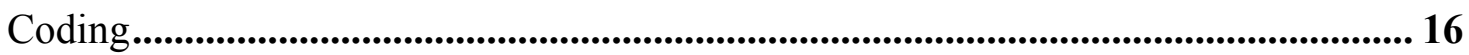

Results and Discussion ....................................................................................................... 17

Experiment 3 ......................................................................................................................... 18



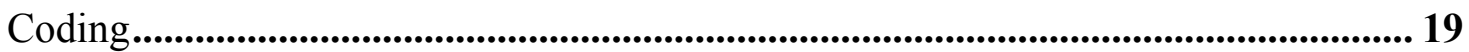

Results and Discussion ......................................................................................................... 20

Experiment 4 .............................................................................................................................. 21

Methods........................................................................................................................................ 21

Coding........................................................................................................................... 23

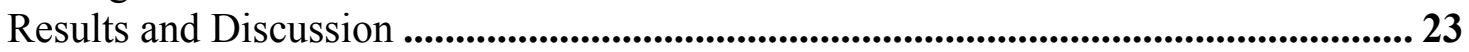



Methods......................................................................................................................... 25

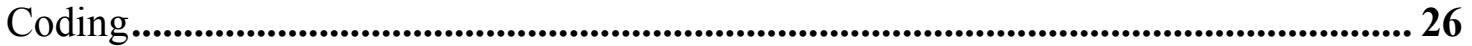

Results and Discussion ..................................................................................... 26

Experiment 6 ....................................................................................................................... 28

Methods............................................................................................................................... 28

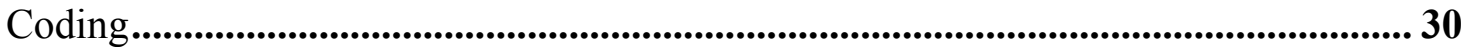

Results and Discussion ................................................................................................. 31

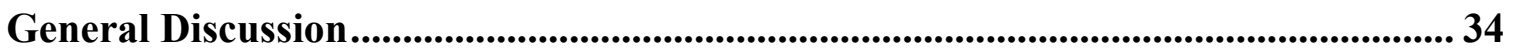

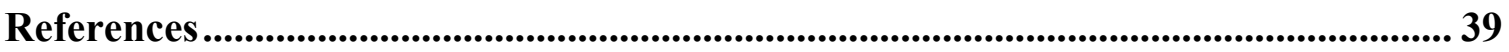




\section{List of Figures}

Figure 1. Examples of positive (fruit) and negative (spiders) stimuli interposed with language speakers (Exp. 1)..........................................

Figure 2. Mean number of trials to habituate observed for English Good, French Good, English Bad and French Bad conditions...........................12

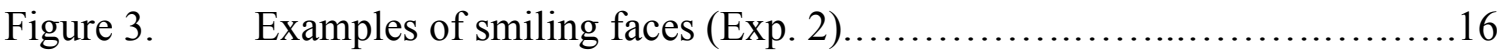

Figure 4. Examples of familiar broken objects (Exp. 3)...........................19

Figure 5. Examples of familiar and novel objects (Exp. 4).........................22

Figure 6. Comparison of mean number of trials to habituate to English and French paired with positive and negative stimuli across experiments...............35 


\section{Acknowledgements}

I would like to offer my thanks to my supervisor, Dr. Andrew Baron, for his

guidance and support. I am grateful for his encouragement and for helping me realize my passion for the work that I am conducting in this field. I would also thank my M.A.

committee members, Dr. Janet Werker and Dr. Frances Chen, for their helpful feedback and insightful comments.

I would also like to thank my family and partner Michael Olson for their continued support and belief in my academic pursuits. 


\section{Introduction}

The persistence of discrimination and group-based conflict among adults across cultures (Allport, 1979; Brewer, 1979; Devine, 1989; Greenwald, Poehlman, Uhlmann, \& Banaji, 2009) as well as the noted difficulty in changing negative out-group attitudes among this cohort (Lai et al., 2014), has led some scholars to question whether intergroup bias is a natural disposition of human psychology. In other words, are people naturally inclined to like those who are similar to others found in their social environment, and despise those who are different? Or, are children "taught" to like those like them and hate the "other"?

Research has demonstrated that children exhibit strong intergroup biases on measures such as peer-preference, moral judgments, explicit valuation, and pro- and antisocial behavior towards both conventional and arbitrary groups, as early as 3-years-ofage (Baron \& Dunham, in press; Dunham, Baron, \& Carey, 2011; Patterson \& Bigler, 2006; Raabe \& Beelmann, 2011; Rutland, Killen, \& Abrams, 2010). Based on the robust evidence that young children have established both implicit and explicit intergroup biases, researchers have turned to infancy to determine whether a positive and/or negative social group bias itself is part of our endowed cognitive capacities.

A review of the infant literature demonstrates that within the first year of life, infants represent categories of people in the domains of race, language, gender and attractiveness, and often exhibit preferences (indicated by longer looking time) for individuals from familiar groups over individuals from unfamiliar groups (Bar-Haim, Ziv, Lamy, \& Hodes, 2006; Kelly et al., 2007; Kelly et al., 2005; Kinzler, Dupoux, \& Spelke, 2007; Quinn, Yahr, Kuhn, Slater, \& Pascalis, 2002; Ramsey, Langlois, Hoss, 
Rubenstein, \& Griffin, 2004).

Although the tendency to establish representations of these social categories and particular preferences emerges early in development, the particular categories represented and the resulting preferences that form are significantly constrained by the child's cultural milieu. A study by Bar-haim et al., 2006) demonstrated that 3-month old African Ethiopian infants raised in a predominantly Caucasian environment (Israel) do not exhibit preferences for own race faces. In contrast, African Ethiopian infants living in Africa (as well as Caucasian infants living in Israel, a predominantly Caucasian environment) do show a clear preference for own-race faces. Therefore, unlike infants living in racially homogenous environments, infants living in racially heterogeneous environments do not exhibit a visual preference for own-race faces. In other words, the strength with which infants exhibit an own-race face preference is heavily influenced by their exposure to other-race faces. In addition, newborn infants do not demonstrate a preference for faces represented by their own-ethnic group (Kelly et al., 2005). Given this evidence, it is important to acknowledge that a preference for own-race faces is learned, and does not spontaneously arise at birth. Together, these studies suggest that selective preferences based on ethnicity is acquired over the first 3 months of life, and heavily depends on the facial input infants receive from their immediate visual environment (Kelly et al., 2005; 2007).

Similar results have been obtained for other social categories. Research shows that infants living in monolingual households exhibit a preference for their native-language over foreign languages (Kinzler et al., 2007). In addition, this result is not limited to Western cultures, as American infants reveal a preference for English over French, but 
the opposite pattern is observed for French infants (exhibiting a preference for French over English). In a further study, 10-month old infants viewed alternating videos of two different Caucasian females. One female spoke to them in a familiar language, while the other in an unfamiliar language. Subsequently, when identical toys were silently presented by both of these speakers in synchrony, infants preferentially accepted toys from the individual who had previously spoken in their native language. This demonstrates that infants not only categorize and show visual preferences for individuals from these groups, but also show prefer to interact with individuals who are from a familiar language group (Kinzler et al., 2007).

Research has also shown that infants' preference to look at categories of gender and facial attractiveness is also mediated by their prior experience with members of these groups. Specifically, whether a child prefers to look at male or female faces is predicted by the sex of their primary caregiver (Quinn et al., 2002). And, infants preference for attractive over unattractive faces appears to be at least partially mediated by their exposure to prototypical faces in the population as infants have been show to exhibit a preference for novel faces that are a composite average of previously viewed faces (Rubenstein, Kalakanis, \& Langlois, 1999; Hoss, Langlois, Pascalis, \& Slater, 2003). Coupled with the findings for race and language, these data suggest that infants' social group preferences is at least partially driven by the degree of familiarity with that group.

Although these studies clearly reveal that infants have a bias towards one group relative to a comparison group, it is unclear whether infants' longer looking time at (or preferential engagement with) a particular stimulus necessarily indicates an evaluative preference. In other words, infants may simply look longer at one (type of) face (e.g., 
native language speaker) over another (e.g., foreign language speaker) because it represents what is more familiar in their social environment. At the same time, infants may not be attributing positivity or negativity toward either individual (or its group). Further, even if infants' preferences are evaluative, it is unclear from forced-choice procedures whether an infant's choice to look at or interact with an individual is due to positivity toward individuals from one group or to negativity toward individuals from the other group. Therefore, addressing the nature of infants' social group preferences is critical to understanding the early developmental roots of intergroup bias, and may reveal which aspects of social group bias are innate.

The present study examined whether infants' social group preferences are based upon a positive evaluation of familiar groups, a negative evaluation of unfamiliar groups, or both. I designed a habituation procedure for infants modeled conceptually after the Implicit Association Test (IAT), used extensively with children and adults to measure evaluative associations with social groups (Baron, 2015; Baron \& Banaji, 2006; Dovidio, Gaertner, Kawakami, \& Hodson, 2002; Fazio \& Olson, 2003; Greenwald, McGhee, \& Schwartz, 1998). The IAT requires participants to quickly classify two types of stimuli using the same response key (or button). For example, members of the ingroup and positive adjectives such as "nice" and "good" will share a response key, while members of the outgroup and negative adjectives such as "mean" and "bad" will share a response key. The IAT is predicated on the logic that evaluatively congruent stimuli (e.g., faces of ingroup members and positive words) are easier (and thus faster) to pair together than evaluatively incongruent stimuli (e.g., faces of outgroup members and the same positive words) (Baron \& Banaji, 2006; Baron \& Dunham, 2011). Therefore, if participants have a positive association with their ingroup, they will be faster to respond when ingroup 
members are paired with positive adjectives.

I chose to focus on language groups in this study because there is robust evidence that infants already represent this category and exhibit visual preferences for native language speakers over foreign language speakers (Kinzler et al., 2007; Kinzler, Shutts, DeJesus, \& Spelke, 2009; Pietraszewski, Cosmides, \& Tooby, 2014; Vouloumanos \& Werker, 2004). Using the logic behind the IAT, I examined infants' ability to pair positive (or negative) stimuli with speakers of a familiar (or unfamiliar) language measured by their differential rates of habituation across such pairings. Substantial work shows that infants can detect congruency among a broad range of stimuli, including matching affective stimuli with evaluatively congruent stimuli. For example, infants fixate longer on the facial expression (e.g., happy, sad, neutral or angry) that is congruent with the valence of the vocal expression that they hear (Spelke, 1976; Walker, 1982). Further, infants' rate of habituation reflects their capacity to detect similarity in objects presented across sequential trials and that successful habituation indicates that they have formed a representation of a category based on the objects presented across those trials (Bornstein, 1985; Bornstein \& Sigman, 1986). Based on this prior work, we reasoned that habituation rates can be used to measure the strength of infants' evaluative associations with a social group.

In Experiment 1, individuals from a familiar language group (English) or unfamiliar language group (French) alternated with either positive stimuli (images of fruit) or negative stimuli (images of spiders). In Experiment 2, I introduced smiling faces as the positive stimuli placed in alternation with the original native/foreign language speakers. 
I hypothesized that if infants have a preference for the familiar language group, then they should habituate faster when viewing English-speaking individuals paired with positive stimuli (images of fruit/smiling faces) compared with children who view Frenchspeakers interposed with the same positive stimuli. Complementarily, if infants have developed a negative evaluation of the unfamiliar language group, then they should habituate at a faster rate to French speakers paired with negative stimuli (images of spiders), than English speakers paired with the same negative stimuli. However, if infants similarly evaluate familiar and unfamiliar language group either positively or negatively, then similar rates of habituation should be observed when English speakers and French speakers are paired with the same evaluative stimulus.

To rule out alternative explanations for my findings, I conducted three additional experiments to ensure that a) infants perceived spiders as sufficiently negative (Experiments 3 and 5), and that b) sheer familiarity did not have a primary effect on infants' habituation rates (Experiment 4). Lastly, I conducted an experiment (Experiment 6) in which infants were habituated to a single kind of action (either prosocial or antisocial) that was performed either by a native or foreign language speaker. Since previous research has already demonstrated infants already perceive prosocial actions as positive, and antisocial actions as negative (Hamlin, Wynn \& Bloom, 2007; Hamlin, Wynn, Bloom \& Mahajan, 2011), this method allowed me to combine the social category from which the actor belonged (native/foreign language speaker) with the valence of their action (positive/negative) in a single trial. Similar to the logic of my first experiment, if infants are associating positive behaviors more strongly with native language speakers, then infants should habituate at a faster rate to a native speaker behaving prosocially than 
a foreign speaker behaving prosocially. On the other hand, if infants associate negative behaviors more strongly with foreign speaking individuals, then they should habituate more quickly to a foreign speaker behaving antisocially than a native speaker performing the same act. 


\section{Experiment 1}

\section{Methods}

Participants

For all experiments in this study, infants were recruited and tested within a local science museum in a sound-proof room dedicated for behavioural science research. A legal guardian provided consent for child participation. According to parental report, all infants in the English conditions were exposed to English at least $80 \%$ of the time, to ensure that English was their primary or familiar language group. None of the infants who participated in the French conditions were exposed to French, thus ensuring that infants were hearing an unfamiliar language. Subjects in all experiments were full-term infants.

In Experiment 1, data from 96 infants (48 females; mean age $=12$ mo 12d, range $=8$ mo 3d-16 mo 29d) who reached habituation were included in the analyses that follow. An additional 33 participants reached the full 30 trials without habituating. These additional participants were distributed similarly across all four conditions. This exclusion number is considered typical given the venue (a local community science centre) in which infants were recruited and tested.

\section{Stimuli}

Puppets speaking either the familiar (English) or an unfamiliar (French) language, alternated with images of fruits or spiders. Participants viewed individuals from a familiar group (English speakers) alternated with either positive stimuli (images of fruit) or negative stimuli (images of spiders), which prior work shows are evaluated as positive and negative respectively (Drewnowski, Mennella, Johnson, \& Bellisle, 2012; LoBue, 
Rakison, \& DeLoache, 2010). In separate conditions, individuals from an unfamiliar group (French speakers) alternated with trials presenting the same images of fruit or spiders as those in the English conditions. The combination of stimuli resulted in four conditions: English Good, French Good, English Bad and French Bad. See Figure 1. English/French Good Conditions

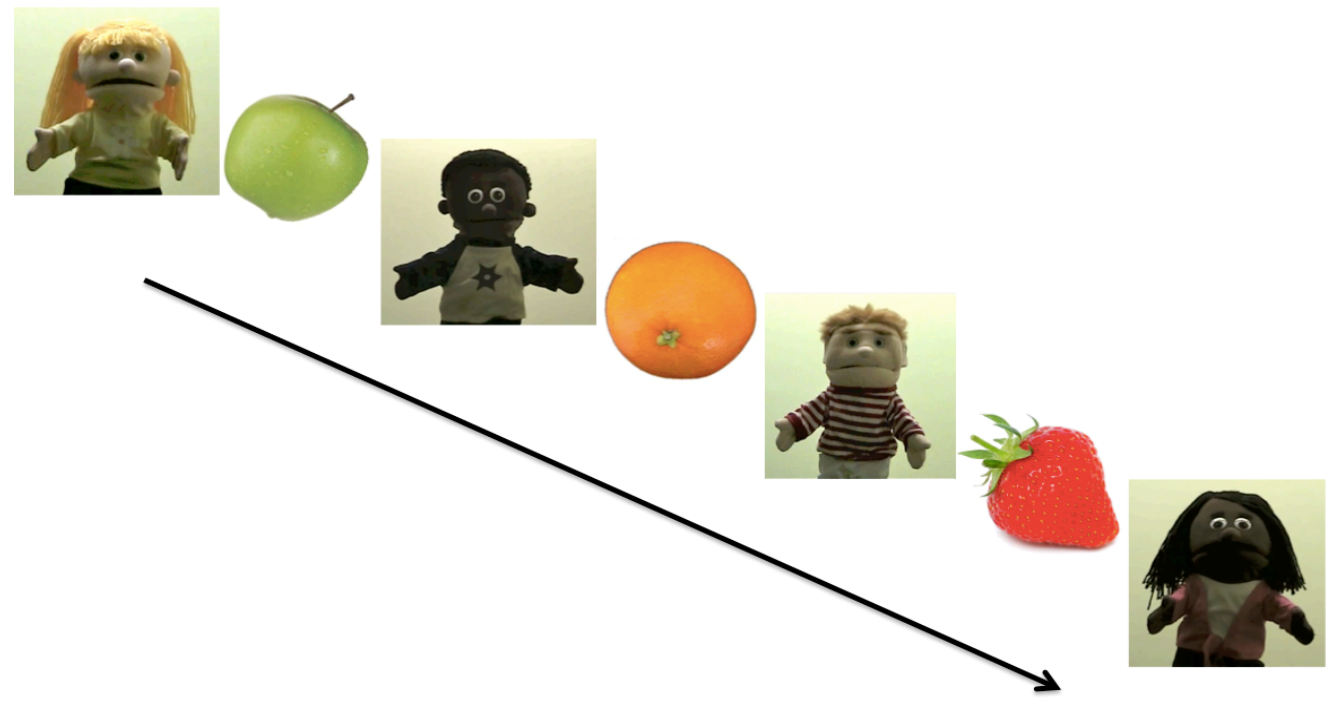

English/French Bad Conditions

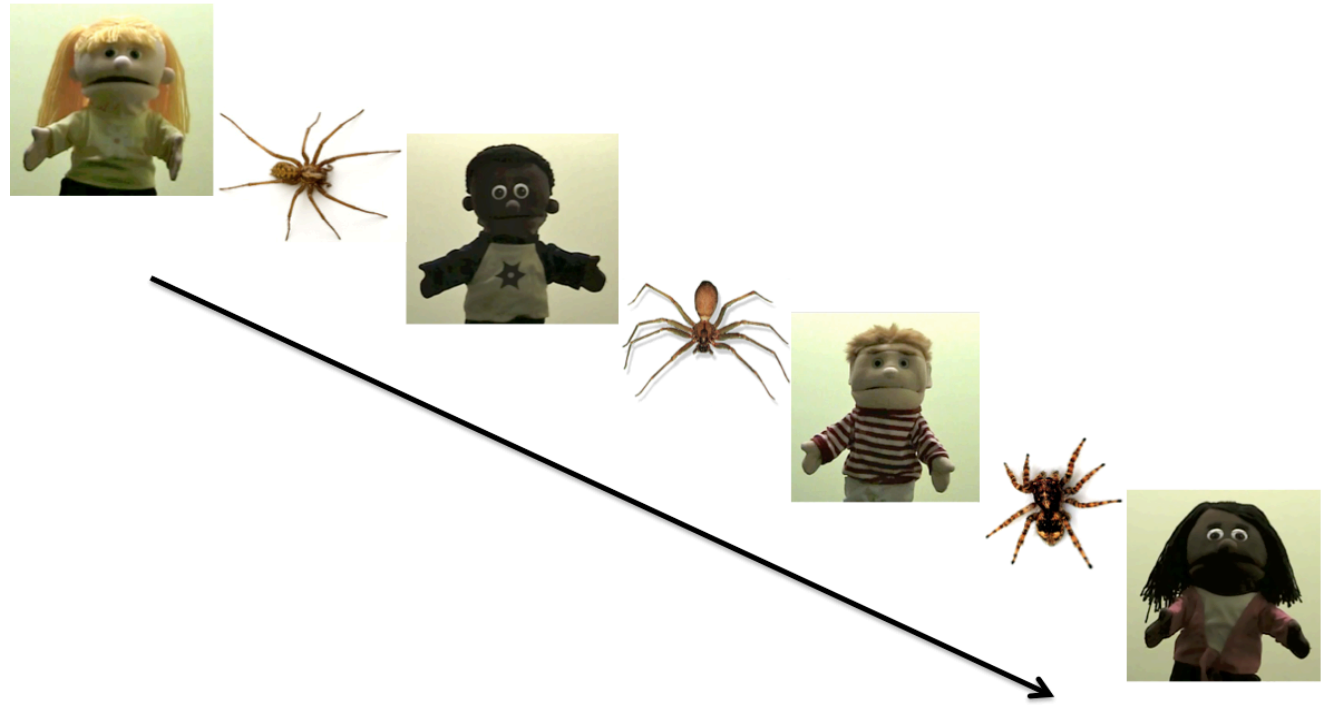

Figure 1. Examples of positive (fruit) and negative (spiders) stimuli interposed with language speakers (Exp. 1). 
Procedure

Infants were randomly assigned to one of four conditions: English Good, French Good, English Bad and French Bad. All participants were tested in a sound proof room, and were positioned on the lap of their caregiver for the duration of the study, approximately $140 \mathrm{~cm}$ from the center of the LCD television screen. To ensure that the caregivers' reactions to the stimuli would not influence the child's behavior, they were instructed to either keep their eyes closed or were asked to wear a pair of blackout glasses for the duration of the study. Caregivers were also asked to remain silent and to not otherwise direct their child's' attention during the course of the study. The experimenter sat adjacent to the infant and caregiver, separated by a distance of approximately 3 feet and hidden behind a black curtain. The experimenter remained behind the curtain and out of the infants' line of sight for the duration of the study.

Once the study began, each trial was preceded by an attention getter, which consisted of a spinning geometric spiral accompanied by a clicking sound. This served the purpose of orienting the child to the center of the screen for the start of each trial. Infants began the study by viewing a video of a single puppet speaking in either English or French for a duration of 10 seconds. After the puppet had finished speaking, the animation froze with the puppet remaining on the screen. There were a total of four puppets introduced throughout the study, and on the TV display were $38 \mathrm{~cm}$ high and 25 cm wide. On alternating trials, infants viewed static images of individual fruits or individual spiders measuring in similar dimensions as the puppets. Total looking duration measured for each trial was recorded from the moment that both the video (or image) was presented and the infants' gaze was fixated on the screen. Termination of each trial by the 
experimenter occurred either when 45 seconds had elapsed, or when an infant looked away for more than 2 seconds. This alternating sequence of trials (puppet video/image of fruit or spider) continued until the infant habituated or until 30 trials passed. Habituation occurred once the mean looking time of the last four trials was equal to or less than half of the average mean looking time for the first four trials. Rate of habituation was the dependent measure.

\section{Coding}

An online coder used the computer program JHab (Casstevens, 2007) to measure infants' looking time in real time. To determine whether each participants' rate of habituation (ie. number of trials to reach habituation) was correctly determined by the online coder, a secondary coder separately analyzed $56 \%$ of the videos over the four conditions. Secondary coders were kept blind to condition (as they did not hear any audio playing) and were also naïve to the hypotheses. The two coders reached agreement on the rate of habituation for $98 \%$ of the participants' videos that were separately analyzed. There was only disagreement with the rate of habituation for one participant (in the French + Fruit condition). The rate of habituation as determined by the online coder was one trial higher than that of the secondary offline coder, which was in the predicted direction. Data from the online coder was used.

\section{Results and Discussion}

Infants' rate of habituation to these sequences was analyzed with a 2 (Language group: Familiar, Unfamiliar) x 2 (Valence object: Positive, Negative) ANOVA, with the number of trials to habituate entered as the dependent variable. The interaction between language group and valence was statistically significant, $F_{(1,95)}=8.11, p=0.005$. See Figure 2. 


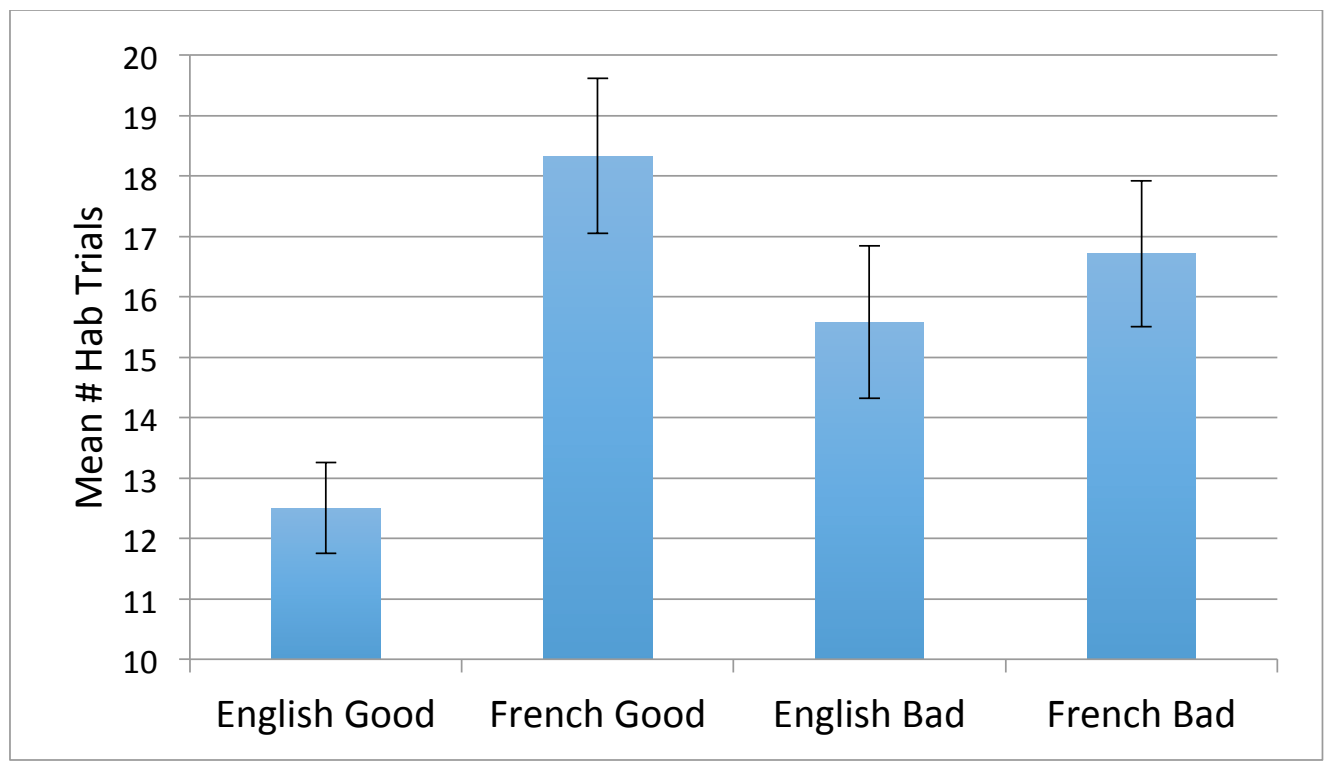

Figure 2. Mean number of trials to habituate observed for English Good, French Good, English Bad and French Bad conditions. A main effect of condition was observed.

Post-hoc comparisons revealed that infants were faster to habituate to English speakers paired with positive stimuli (Mean \# trials to Hab $=12.08$ ) than French speakers paired with positive stimuli (Mean \# trials to Hab $=19.67$ ), $t(46)=-5.23, p<.001, d=$ $1.54,95 \%$ CI $[-10.50,-4.67]$ demonstrating that infants have established a greater positive association with English speakers compared to French speakers.

In addition, if infants feel positively towards familiar language speakers in general (and associate positivity more readily with familiar speakers than negativity), they will be slower to habituate to English paired with negative stimuli compared with English paired with positive stimuli. However, if infants are neutral towards familiar language groups, than there should not be a significant difference in rates of habituation for English paired with positive or negative stimuli. Post-hoc analyses revealed that infants were significantly slower to habituate to English speakers paired with negative stimuli (Mean \# 
trials to $\mathrm{Hab}=15.25)$, compared with positive stimuli (Mean \# trials to Hab $=12.08)$ $t(46)=-2.25, p=.03, d=0.66,95 \%$ CI $[-6.01,-0.33]$ further underscoring that infants have established a greater positive association with familiar (English) speakers and feel more positively towards the familiar group. Taken together, these data suggest that a positive evaluation of the familiar group has already formed within the first year of life.

To address whether infants feel negatively towards the unfamiliar language group, we conducted post-hoc comparisons to determine whether infants habituated more quickly to French speakers paired with negative or positive stimuli. If infants feel more negatively towards the unfamiliar language group, then they should habituate at a faster rate to French paired with negative stimuli compared with French paired with positive stimuli. However, if they feel neutral towards the unfamiliar language group, then there should not be a significant difference in rates of habituation to French paired with positive or negative stimuli. A post-hoc analysis revealed that infants habituated to French speakers paired with negative stimuli (Mean \# trials to Hab $=16.46$ ) at a slightly faster rate than French speakers paired with positive stimuli (Mean \# trials to Hab = $19.67), t(46)=1.85, p=.07, d=0.54,95 \% \mathrm{CI}[-0.29,6.71]$, suggesting that infants associate unfamiliar language speakers with negativity more readily than positivity. However, this result is only marginally significant. While this may be interpreted as infants demonstrating greater negativity towards unfamiliar speakers, a crucial comparison between English and French speakers paired with spiders reveals that infants are not evaluating French speakers more negatively than English speakers. This is because infants were similarly quick to habituate to English speakers paired with negative stimuli (Mean \# trials to Hab = 15.25) compared with French speakers paired with 
negative stimuli (Mean \# trials to $\mathrm{Hab}=16.46), t(46)=-0.71, p=.48, d=0.21,95 \%$ CI [$4.64,2.23]$. Taken together, these data suggests that infants do not readily associate negativity with either French or English speakers.

To investigate the possibility of age effects with our sample, we ran an ANOVA with the number of trials to habituate entered as the dependent variable and 3 between subjects factors: age group, language group (English, French) and object type (positive, negative) were entered. We computed separate ANOVAs for age group split into halves (mean split) and age split into quartiles. There was no main effect of age for either age grouping (split half or quartiles, $p \mathrm{~s}>.30$ ). Although there was a significant two-way interaction between language group and age split into halves $(p=.017)$, there was critically no significant interaction between language group, object type or age $(p=.43$ for age split into halves; $p=.40$ for age split into quartiles). Thus, the main findings reported in Experiment 1 were not driven by a subgroup of our sample based on participants' age. 


\section{Experiment 2}

To conceptually replicate our findings of the English Good and French Good conditions, we conducted a second experiment (Experiment 2) using the same English and French speaking puppets from Experiment 1, but interposed happy facial expressions instead of images of fruits. In infants indeed evaluate the familiar language group more positively than the unfamiliar language group, then they should habituate more quickly to English paired with positive stimuli (smiling faces) compared to French paired with positive stimuli.

\section{Methods}

Participants

Data from 48 infants ( 24 females; mean age $=13$ mo $8 \mathrm{~d}$, range $=7$ mo $30 \mathrm{~d}-16$ mo 28d) who reached habituation were included in the analyses. Six additional infants were not included because they did not reach habituation after the full 30 trials. These additional participants were distributed similarly across both conditions. Stimuli

The same English and French speaking puppets from Experiment 1 were alternated with an alternative class of positive stimuli; smiling faces. The colors of the smiling faces were chosen to be green, orange and red to maintain consistency with the color of the fruits used in Experiment 1 (green apple, orange and strawberry). See Figure 3. 

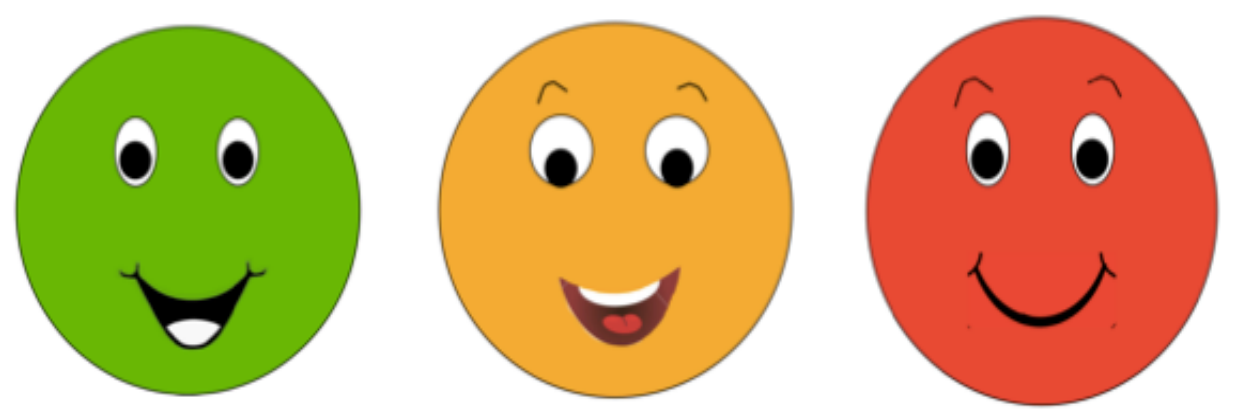

Figure 3. Examples of smiling faces.

When the image of the face initially appeared on the screen, the corners of the mouth were animated to turn upwards into an intense smile, and the pupils moved slightly upwards to reveal a happy expression. This was done to ensure that infants focused on the expression itself and would appropriately perceive it as smiling and happy. The combination of the stimuli resulted in two conditions: English Happy and French Happy. Procedure

Participants were randomly assigned to one of two conditions: English Happy and French Happy. All procedures were identical to Experiment 1. As with Experiment 1, rate of habituation was the dependent measure.

\section{Coding}

Coding procedures were identical to those used in Experiment 1. To determine whether each participants' rate of habituation (ie. number of trials to reach habituation) was correctly determined by the online coder, a secondary coder separately analyzed $29 \%$ of the videos over the two conditions. The two coders reached agreement on $100 \%$ of the videos that were separately analyzed. 


\section{Results and Discussion}

As in Experiment 1, we observed that infants were faster to habituate to English speakers paired with smiling faces (Mean \# trials to $\mathrm{Hab}=15.21$ ) compared with French speakers paired with smiling faces (Mean \# trials to $\mathrm{Hab}=18.67$ ), $t(46)=-2.46, p=.018$, $d=0.73,95 \% \mathrm{CI}[-6.29,-0.63]$. These results reveal that infants have greater positivity towards the familiar group (English).

To investigate the possibility of age effects with our sample, we ran an ANOVA with the number of trials to habituate entered as the dependent variable and 3 between subjects factors: age group, language group (English, French) and object type (positive) were entered. We computed separate ANOVAs for age group split into halves (mean split) and age split into quartiles. No main effect of age was observed for either age grouping (split halves or quartiles, $p s>.21$ ). In addition, there was no significant interaction between language group and age split into quartiles $(p=.24)$. A marginally significant interaction between language group and age split into halves $(p=.07)$ was found. Once again, the main findings reported in Experiment 2 were not driven by a subgroup of our sample based on participants' age. 


\section{Experiment 3}

In Experiment 1, we did not observe a significant difference in rate of habituation to English and French paired with negative stimuli (spiders). However, it is unclear whether infants truly do not evaluate either familiar or unfamiliar language speakers negatively at this age, or whether this result was obtained due to a methodological limitation (ie. infants did not perceive spiders as negative). Experiment 3 aimed to conceptually replicate the English/French negative conditions of Experiment 1 with a different class of stimuli also known to evoke negativity in young children, namely broken familiar objects (Kagan, 1981; Kochanska, Casey, \& Fukumoto, 1995). If infants negatively evaluate unfamiliar language speakers, then infants in the present experiment should habituate more quickly to French speakers paired with broken familiar objects compared to English speakers paired with broken familiar objects. However, if infants do not show a significant difference in rates of habituation between French speakers paired with broken objects or English speakers paired with broken objects, it is unlikely that an unfamiliar language group is being evaluated more negatively than the familiar language group.

\section{Methods}

Participants

Data from 48 infants ( 24 females; mean age $=12$ mo $24 \mathrm{~d}$, range $=7$ mo $28 \mathrm{~d}-16$ mo 29d) who reached habituation were included in the analyses. An additional 19 infants participated in the study, but were excluded from the analyses because they did not reach habituation after the full 30 trials. These additional participants were distributed similarly across both conditions. 
Stimuli

The same English and French speaking puppets from Experiment 1 were used, but the images of spiders from Experiment 1 were replaced with static images of broken familiar objects (e.g., torn teddy bear, broken chair, and broken cup). See Figure 4.

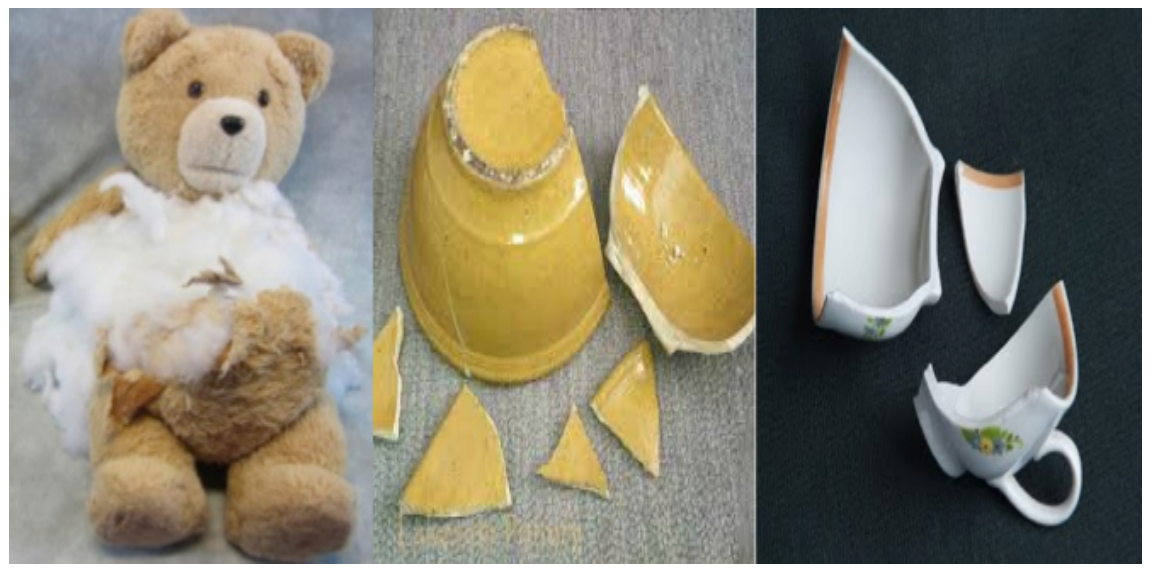

Figure 4. Examples of familiar broken objects.

The combination of stimuli resulted in two conditions: English Broken Objects and French Broken Objects.

Procedure

Participants were randomly assigned to one of two conditions: English Broken Objects and French Broken Objects. All procedures were identical to Experiment 1. As with Experiments 1 and 2, rate of habituation was the dependent measure.

\section{Coding}

Coding procedures were identical to Experiment 1 . For the $44 \%$ of videos that were separately analyzed by a secondary offline coder (over the two conditions), the two coders reached agreement on $95 \%$ of the videos. There was only disagreement with the rate of habituation for one participant (in the English Broken Objects condition). The rate 
of habituation as determined by the online coder was one trial higher than that of the secondary offline coder. Data from the online coder was used.

\section{Results and Discussion}

We replicated our findings from the English/French Bad conditions in Experiment 1, in which no significant difference was observed in infants' rate of habituation in the English + Broken Objects condition (Mean \# trials to Hab =17.29) compared to the French + Broken Objects condition (Mean \# trials to Hab $=16.88),(p=.82)$. Once again, this suggests no negativity is held toward French speakers. 


\section{Experiment 4}

Experiments 1-3 revealed that infants' language group preferences are based upon a positive evaluation of the familiar language group without a corresponding negative evaluation of an unfamiliar language group. However, it is possible that infants may have habituated faster in the English+Good conditions because both classes of stimuli (English speakers and images of fruit/smiling faces) might be more familiar to infants relative to the other classes of stimuli (French speakers and images of spiders). A partial response to this concern is that we found no overall difference in looking time at images of fruits or spiders $\left(M_{F r}=5.23\right.$ seconds, $S D=1.95 ; M_{S p}=5.76$ seconds, $\left.S D=2.62\right), \mathrm{t}(94)=-1.12, p$

$=.26$ ). Nonetheless, to more directly address this concern, Experiment 4 assessed the potential effect of stimulus familiarity on infants' rate of habituation. Specifically, infants were habituated to English or French speakers (between-subjects) paired with images of either familiar stimuli (familiar vehicles) or novel stimuli (obscure mechanical parts) borrowed from past studies of word learning for familiar and unfamiliar objects with infants and toddlers (Halberda, 2006). We reasoned that if infants habituated faster to English paired with fruits (English Good condition in Experiment 1) because both English and fruits are more familiar than French, then infants should habituate more quickly to English paired with familiar objects compared to English paired with novel objects (or to French paired with familiar objects).

\section{Methods}

Participants

Data from 96 infants (48 females; mean age $=12$ mo 13d, range $=7$ mo 29d -16 mo 21d) who reached habituation were included in the analyses. Data from an additional 
29 infants tested were not included because they did not habituate after the full 30 trials. These additional participants were distributed similarly across all four conditions.

Stimuli

The same English and French speaking puppets from Experiment 1 were used. However, images of fruits and spiders from Experiment 1 were replaced with images of familiar and novel objects respectively. See Figure 5.
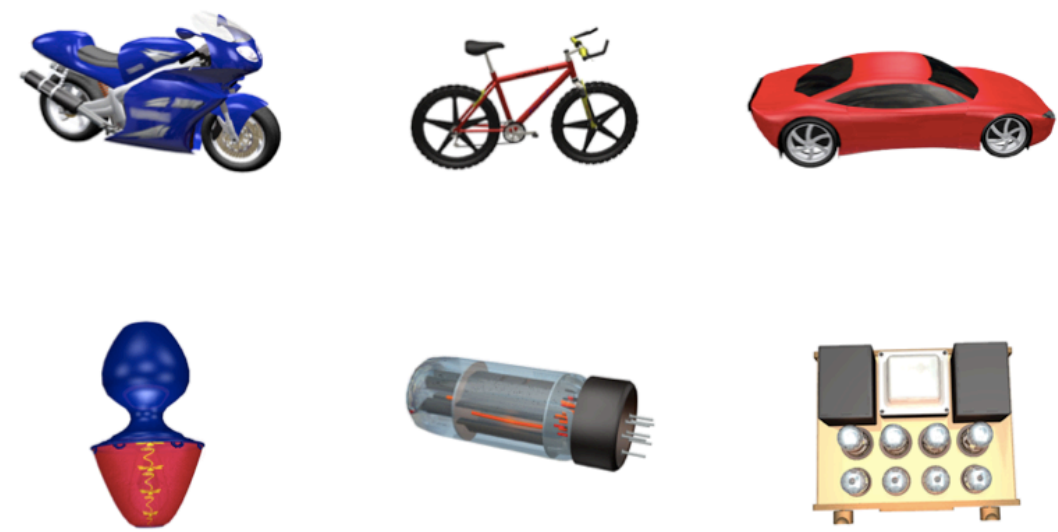

Figure 5. Examples of familiar (top row) and novel objects (bottom row).

The combination of stimuli resulted in four conditions: English Familiar, French Familiar, English Novel and French Novel.

Procedure

Participants were randomly assigned to one of four conditions: English Familiar, French Familiar, English Novel and French Novel. All procedures were identical to Experiment 1. As with Experiments 1-3, rate of habituation was the dependent measure. 


\section{Coding}

Coding procedures were identical to those used in Experiment 1 . For the $37 \%$ of videos (over 4 conditions) that were separately analyzed by a secondary offline coder, the two coders reached agreement on $98 \%$ of the videos. There was only disagreement with the rate of habituation for one participant (in the English Novel condition). The rate of habituation as determined by the online coder was one trial higher than that of the secondary offline coder. Data from the online coder was used.

\section{Results and Discussion}

Contrary to this prediction, a 2 (Language group: Familiar, Unfamiliar) x 2 (Object type: Familiar, Unfamiliar) ANOVA with the number of trials to habituate entered as the dependent variable revealed no main effect of language $(p=.70)$, object type $(p=.20)$, or an interaction between these two terms $(p=.34)$. For instance, the mean number of trials to habituation for English Familiar $(M=15.08)$ and French Familiar $(M=15.75)$ did not differ $(p=.69)$, and the mean number of trials to habituation for English Novel $(M=17.71)$ and French Novel $(M=16.13)$ did not differ either $(p=.34)$. This suggests that infants are not simply habituating faster to familiar pairings in general. Consequently, familiarity alone is unlikely to account for the results reported in Experiment 1. In addition, we analyzed the average looking towards English and French puppets as well as average looking towards familiar and unfamiliar objects. No difference was observed in baseline interest toward either English or French speakers $\left(\mathrm{M}_{\mathrm{En}}=\right.$ $15.90 \mathrm{~s}, \mathrm{SD}=7.0$ and $\left.\mathrm{M}_{\mathrm{Fr}}=17.11 \mathrm{~s}, \mathrm{SD}=6.22, t(94)=-0.89, p=.38\right)$ or toward Novel or Familiar objects $\left(\mathrm{M}_{\mathrm{Fam}}=6.67 \mathrm{~s}, \mathrm{SD}=4.34\right.$ and $\mathrm{M}_{\mathrm{Nov}}=5.58 \mathrm{~s}, \mathrm{SD}=3.06, t(94)=1.42, p=$ .16). Therefore, it is unlikely that unfamiliar stimuli (French speakers or novel objects) 
lead to longer looking-time in general which may potentially contribute to more difficulty habituating to these stimuli.

Once again, to investigate the possibility of age effects with our sample, we ran an ANOVA with the number of trials to habituate entered as the dependent variable and 3 between subjects factors: age group, language group (English, French) and object type (Familiar, Novel) were entered. We computed separate analyses for age group split into halves (mean split), and age split into quartiles. No main effect was observed for either age groupings (split halves and quartiles, $p \mathrm{~s}>.52$ ). Furthermore, there was no significant 3 -way interaction between language group, object type and age group $(p=.17$ for age split into halves; $p=.83$ for age split into quartiles). Thus, the main findings reported in Experiment 4 were not driven by a subgroup of our sample based on participants' age. 


\section{Experiment 5}

To further ensure there was not a methodological limitation in our ability to measure associations with negativity in our paradigm, a fifth experiment attempted to address the perceived valence of the stimuli that had been paired with language groups in Experiments 1 and 3, by directly assessing the strength of the association between them. Specifically, we assessed whether infants view spiders and broken objects as negative, and thus as different from fruits. We reasoned that if infants do perceive spiders and broken objects as negative (Kagan, 1981; LoBue et al., 2010), they should habituate faster to a sequence of trials in which images of spiders alternate with images of broken objects, compared with a sequence of trials in which images of fruit alternate with images of broken objects.

\section{Methods}

Participants

Data from 48 infants $(24$ females; mean age $=11$ mo $29 \mathrm{~d}$, range $=8$ mo $2 \mathrm{~d}-16$ mo 19d) who reached habituation were included in the analyses. Another 8 infants participated, but were excluded from the analyses because they did not habituate after viewing the full 30 trials. These additional participants were distributed similarly across all four conditions.

Stimuli

The same fruit and spider images from Experiment 1, as well as the images of broken familiar objects from Experiment 4 were used. The combination of stimuli resulted in two conditions: Broken Objects + Spiders, and Broken Objects + Fruit. Each infant only participated in one condition (between- subjects). 
Procedure

Participants were randomly assigned to one of two conditions: Broken Objects + Spiders and Broken Objects + Fruit. The procedure for Experiment 5 was very similar to that for Experiments 1-4. However, infants were only presented with static images during each trial since this experiment did not specifically examine evaluative associations with language groups. As with Experiments 1-4, rate of habituation was the dependent measure.

\section{Coding}

Coding procedures were identical to Experiment 1 . For the $54 \%$ of videos that were separately analyzed by a secondary offline coder (over the two conditions), the two coders reached agreement on $95 \%$ of the videos. There was only disagreement with the rate of habituation for one participant (in the Broken Object + Fruit condition). The rate of habituation as determined by the online coder was one trial higher than that of the secondary offline coder, which was in the predicted direction. Data from the online coder was used.

\section{Results and Discussion}

As predicted, infants were faster to habituate to broken objects paired with spiders $($ Mean \# trials to $\mathrm{Hab}=10.00)$ compared to when broken objects were paired with images of fruit (Mean \# trials to $\mathrm{Hab}=13.71), t(46)=2.49, p=.016, d=0.74,95 \% \mathrm{CI}[0.72$, 6.70]. We interpret this result as demonstrating that infants evaluate broken objects and spiders negatively.

Moreover, the finding that infants habituated faster to spiders paired with broken objects further speaks against the concern that less familiar object categories in general 
will lead to slower rates of habituation (e.g., when French was paired with spiders, novel objects or broken objects). If this were a valid concern we would have expected infants to habituate slower to these less familiar object categories in Experiment 5. These findings also rule against the possibility that infants experienced difficulty habituating to arguably visually more complex stimuli (e.g., spiders, broken objects) because our fastest rate of habituation was observed when infants were presented with these stimuli (Mean \# trials = 10.00) compared with when these stimuli were paired with English and French speakers (Mean \# trials > 15). In sum, the reported failure to detect evidence of negative evaluations of unfamiliar language groups (as reported in Experiments 1 and 3) likely suggests that negative evaluations of social groups may develop later than positive evaluations of such groups. 


\section{Experiment 6}

Finally, to obtain a secondary measure of infants' positive and negative evaluations of language groups, Experiment 6 examined whether infants have different expectations about whether familiar and unfamiliar language speakers will engage in prosocial (helping) or antisocial (stealing) behaviour. In other words, do infants expect familiar and unfamiliar language speakers to be nice, or mean? Following Hamlin and colleagues who showed that similar aged infants prefer prosocial agents over antisocial

agents (Hamlin \& Wynn, 2011), we showed infants the same prosocial or antisocial event (between-subjects), but manipulated whether the actor spoke English or French prior to engaging in that behaviour. If infants expect familiar language speakers to be nicer than unfamiliar language speakers, we would expect infants to habituate at a faster rate to English speaking individuals behaving prosocially, compared to French speaking individuals behaving prosocially.

\section{Methods}

Participants

Data from 96 infants (45 females; mean age $=8$ mo $19 \mathrm{~d}$, range $=6$ mo-12 mo) who reached habituation were included in the analyses.

\section{Stimuli}

Infants saw two puppets on stage (the same white male puppet from previous studies), with one puppet wearing a blue and yellow shirt, and the other wearing a red and white striped shirt. Both of the puppets were identical with the exception of shirt color. Specifically, infants saw two puppets on stage, but only one puppet introduced himself verbally in either English or French. The puppet on the infants' left hand side (The Actor) 
spoke in either English or French saying "Hi, look at me. Watch what I'm going to do. Are you ready?" Infants then viewed the "Ball show" (see Hamlin \& Wynn, 2011) in which the puppet on the right (the Protagonist, who remains silent) is seen playing with a ball, repeatedly jumping up and down while bouncing and catching the ball. After the third bounce, the Protagonist drops the ball, and it rolls to the opposite side of the stage. The ball is retrieved by the puppet who had previously spoken in a native or foreign language. In one instance, the speaker gives the ball back to the Protagonist (Nice condition). In another instance, the speaker does not return the ball to the Protagonist and instead takes the ball offstage (Mean Condition). This resulted in four conditions: English Nice, English Mean, French Nice, and French Mean.

Procedure

Participants were randomly assigned to one of four conditions: English Nice, English Mean, French Nice, and French Mean. All participants were tested in a sound proof room, and were positioned on the lap of their caregiver for the duration of the study, approximately $140 \mathrm{~cm}$ from the center of the LCD television screen. To ensure that the caregivers' reactions to the stimuli would not influence the child's behavior, they were instructed to either keep their eyes closed or were asked to wear a pair of blackout glasses for the duration of the study. Caregivers were also asked to remain silent and to not otherwise direct their child's' attention during the course of the study. The experimenter sat adjacent to the infant and caregiver, separated by a distance of approximately 3 feet and hidden behind a black curtain. The experimenter remained behind the curtain and out of the infants' line of sight for the duration of the study. 
Once the study began, each trial was preceded by an attention getter, which consisted of a spinning geometric spiral accompanied by a clicking sound. This served the purpose of orienting the child to the center of the screen for the start of each trial. Infants began the study by viewing two puppets on stage. The puppet on the infants' left hand side (The Actor) spoke in either English or French. Then, infants saw the Protagonist complete the "Ball show" (see Hamlin \& Wynn, 2011). After the Actor ran offstage, and the Protagonist turned back towards the front, the animation froze. Events lasted $\approx 21$ seconds for the English/French nice sequences, and $\approx 19$ seconds for the English/French mean sequences. Looking time was measured at the point in which the animation froze, and continued until the infant either looked away for 2 consecutive seconds, or until 30 seconds elapsed as determined by an online coder and the computer program JHab (Casstevens, 2007). Infants continued to see the same initial sequence for subsequent trials, until they reached habituation.

\section{Coding}

An online coder used the computer program JHab to measure infants' looking time in real time. Looking time was measured at the point in which the action paused, and continued until the infant either looked away for 2 consecutive seconds, or until 30 seconds elapsed. To determine whether each participants' rate of habituation (ie. number of trials to reach habituation) was correctly determined by the online coder, a secondary coder separately analyzed $25 \%$ of the videos over the four conditions. Secondary coders were kept blind to condition (as they did not hear any audio playing) and were also naïve to the hypotheses. The two coders reached agreement on the rate of habituation for $96 \%$ of the participants' videos that were separately analyzed. There was only disagreement 
with the rate of habituation for one participant (in the French Nice Condition). The rate of habituation as determined by the online coder was one trial higher than that of the secondary offline coder, which was in the predicted direction. Data from the online coder was used.

\section{Results and Discussion}

Our data revealed that infants were faster to habituate to English speakers behaving prosocially (Mean \# trials to Hab $=7.04$ ) compared to French speakers behaving prosocially (Mean \# trials to $\mathrm{Hab}=9.46$ ), $t(46)=-3.34, p=.002, d=0.98,95 \%$ CI $[-3.87$, - 0.96]. While infants appear to expect familiar language group members to be nice, they do not hold this expectation for unfamiliar language group members. This is consistent with the results found in Experiments 1 and 2, in which infants habituated more quickly to English speakers paired with positive images than French speakers paired with positive images.

To address whether infants feel more positive in general towards the familiar language group, we analyzed whether infants would habituate at different rates to English speakers being nice, compared to English speakers being mean. If infants feel positively towards familiar (English) speakers, then they should look longer when an English speaker is mean. However, if infants feel neutrally towards the familiar language group, then we would expect them to habituate at similar rates to English speakers being nice or mean. We found that infants were significantly slower to habituate to English speakers behaving antisocially (Mean \# trials to Hab $=8.50$ ) compared to English speakers behaving prosocially (Mean \# trials to Hab $=7.04$ ), $t(46)=-2.67, p=.01, d=0.79,95 \%$ CI $[-2.56,-0.36]$. This suggests that infants have the expectation that familiar language 
group members will be nice, and are more surprised when familiar group members are mean.

In addition, there was no significant difference observed in the rate of habituation between English and French speakers performing antisocial behaviors, as infants were similarly quick to habituate to English speakers behaving antisocially (Mean \# trials to $\mathrm{Hab}=8.5)$ as French speakers behaving antisocially (Mean \# trials to Hab $=8.17),(p=$ .61). This consistent with the results found in Experiments 1 and 3, in which infants did not habituate faster to English or French speakers paired with negative images.

Although we did not find that infants readily associated negativity with either familiar or unfamiliar language speakers in Experiments 1 and 3, we were interested in determining whether infants have developed expectations about the behaviors that unfamiliar language speakers should (or should not) engage in. If infants expect French speakers to be mean, then we would expect them to habituate more quickly to French speakers behaving antisocially than French speakers behaving prosocially. Thus, we compared infants' rates of habituation to French speakers behaving prosocially and antisocially. However, we did not observe a significant difference in the rate of habituation between French speakers performing antisocial (Mean \# trials to Hab $=8.17$ ) or prosocial behaviors (Mean \# trials to Hab $=9.46),(p=.11)$ suggesting that infants do not necessarily expect French speakers to be mean.

The present results suggest that in the context of social groups, infants reason differently about the same prosocial and antisocial behaviors; expecting speakers of a familiar language to engage in a prosocial behavior over speakers of an unfamiliar 
language, while not having formed expectations about which groups will perform antisocial behaviors. 


\section{General Discussion}

Although numerous studies claim that infants have social group preferences based on such properties as their spoken language, race, gender, and attractiveness, the nature of this preference was unclear because these previous demonstrations relied on methods that obscured whether preference was simply a proxy for familiarity (e.g., looking longer to things you recognize) or whether preference indeed entailed evaluative content (e.g., looking longer to things you like). Moreover, previous work neglected to explain whether preferences, if they entail evaluative content, are based upon a positive evaluation (e.g., looking longer to $\mathrm{X}$ compared with $\mathrm{Y}$ because you like $\mathrm{X}$ ), a negative evaluation (e.g., looking longer to $\mathrm{X}$ compared with $\mathrm{Y}$ because you dislike $\mathrm{Y}$ ), or both (like $\mathrm{X}$ and dislike $\mathrm{Y})$.

Our study is the first to demonstrate that within the first year of life, infants do hold evaluative associations regarding social groups, and that positivity toward individuals from familiar social groups emerges independently and prior to negativity towards individuals from unfamiliar social groups. Specifically, infants think that speakers of a familiar language (English) are good relative to speakers of a foreign language (French). However, our data did not reveal a corresponding negative evaluation of speakers of an unfamiliar language. See Figure 6. 


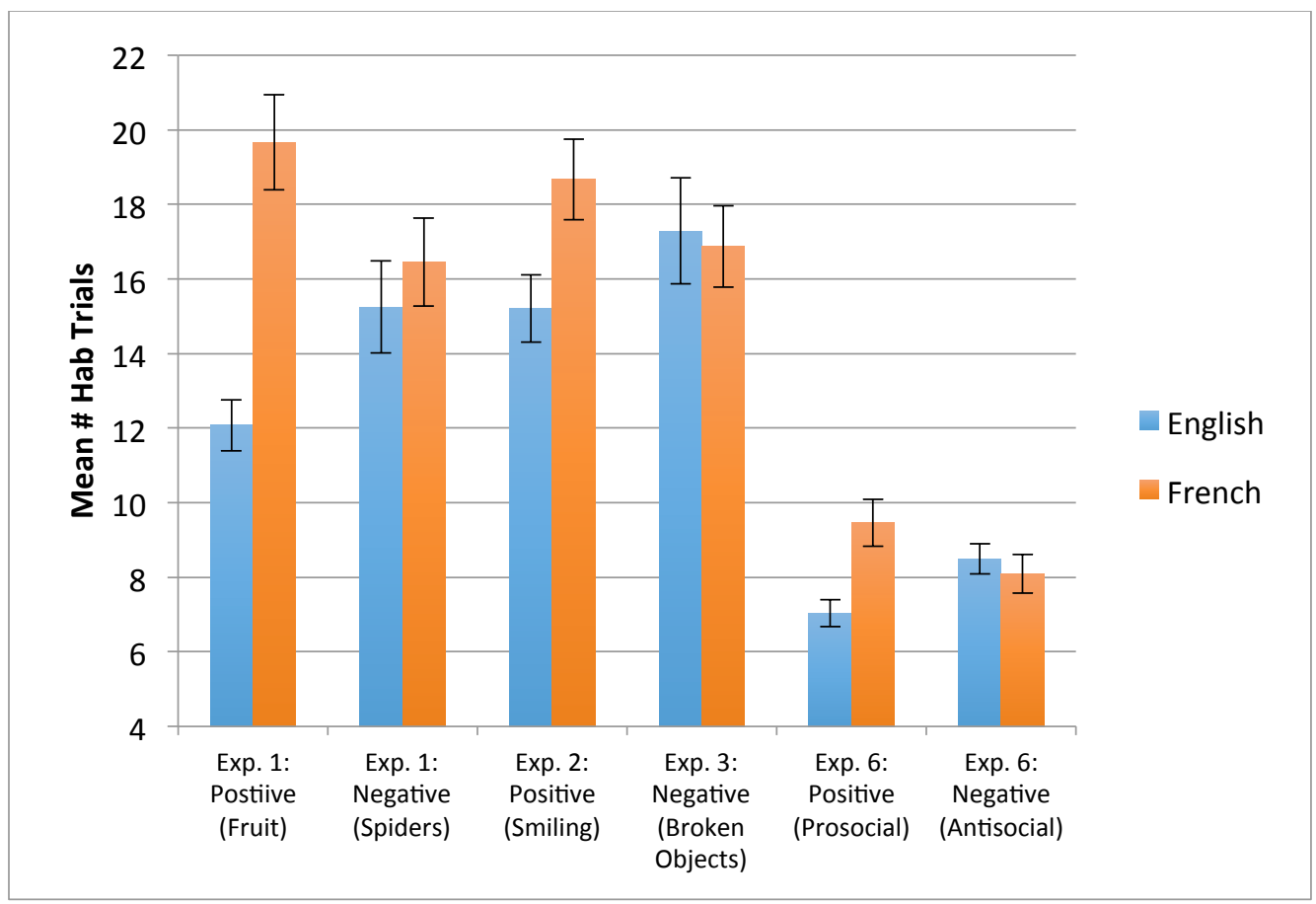

Figure 6. Comparison of mean number of trials to habituate to English and French paired with positive and negative stimuli across experiments.

It is unlikely that this absence of a negativity bias is due to a cognitive limitation of infants' ability to form negative evaluations of individuals or of categories more generally. Indeed, across numerous paradigms, Hamlin and colleagues have demonstrated that 3 and 6-month-old infants are capable of evaluating an antisocial agent negatively (Hamlin, Wynn, \& Bloom, 2007; Hamlin, Wynn, \& Bloom, 2010). And yet, data from Experiment 6 shows that when using the same events as Hamlin and colleagues in the context of social groups rather than individuals, infants fail to demonstrate a negative evaluation of one group relative to the other. Finally, data from Experiment 5 show that infants are capable of forming negative evaluations of two distinct categories (broken objects and spiders). Therefore, even though infants can form negative evaluations of individuals and object categories, it may not be the case that they are yet able or willing 
to form negative evaluations of social groups, at least with regards to speakers of the same language.

These data have several implications for theories of intergroup bias. First, if intergroup bias begins as a positivity bias toward familiar social groups, then future research needs to address how these initial representations begin to engender negativity toward unfamiliar groups. One route for such process may have to do with the development of a social self, which might lead to the conceptualization of familiar and unfamiliar individuals as ingroup and outgroup respectively. Indeed, no study to date has successfully demonstrated that infants actually represent social groups as ingroups and outgroups, as this is often confounded with the group's status as familiar and unfamiliar, respectively.

Although the studies reported here do not address the source of this early evaluative preference, the broad literature documenting the effects of familiarity on preference formation suggests that such preferences may be rooted in a bias to positively evaluate familiar stimuli in general. Indeed, the work of Zajonc has long demonstrated that familiarity leads to liking (Zajonc, 1968, 2001). While familiarity may breed liking (and positive evaluations of the familiar), it does not follow that familiarity necessitates a positive evaluation of that object (category) nor does it further our understanding of the origins of negative attitudes toward unfamiliar (out) groups. Determining what mechanisms lead infants to form evaluative representations of social groups will help reveal which mechanisms of intergroup cognition may be innate.

A second general implication of these findings for future research is to explore whether the absence of negativity toward unfamiliar groups is specific to language 
groups, or whether it is a general feature of infants' intergroup attitudes. Interestingly, a finding such as this in infancy (in which negative attitudes towards a social group is absent) is consistent with what is observed among preschool and young school-aged children (Aboud, 2003), and recent findings reveal that positive attitudes toward ingroup members seem to arise prior to negative attitudes toward outgroup members (Buttelmann \& Böhm, 2014). These findings raise the possibility that implicit and explicit intergroup bias may share a similar trajectory very early in childhood, despite their clear divergence between ages 6-10 (Baron \& Banaji, 2006).

Finally, our findings shed light on the potential evolutionary functions of intergroup biases. In particular, according to certain evolutionary psychologists (Kurzban, Tooby, \& Cosmides, 2001; Pietraszewski et al., 2014), natural selection likely led to the development of cognitive processes specialized for reasoning about social alliances. These processes would have helped humans identify potential collaborators (Kurzban et al., 2001), competitors (McDonald, Navarrete, \& Van Vugt, 2012), or both (Choi \& Bowles, 2007). Motivationally, a drive to identify potential collaborators would be manifested in ingroup favouritism whereas a drive to identify potential competitors would be manifested in outgroup derogation. Arguably, however, at the intergroup level, the formation of collaborative groups would be a prerequisite for the emergence of competition with outgroups. Our findings that infants' positive evaluations of individuals who are part of their social group emerge before negative evaluations of individuals from other social group, is strikingly consistent with this argument. Thus, together with research on both humans (De Dreu, Greer, Van Kleef, Shalvi, \& Handgraaf, 2011; Rand, Dreber, Ellingsen, Fudenberg, \& Nowak, 2009) (e.g., oxytocin promotes ethnocentrism) 
and their primate ancestors (Tomasello \& Vaish, 2013) (e.g., chimpanzees only cooperate with ingroup members and engage in social grooming), the current findings are consistent with the notion that intergroup biases are a product, first and foremost, of the need to form cooperative alliances. 


\section{References}

Aboud, F. E. (2003). The formation of in-group favoritism and out-group prejudice in young children: Are they distinct attitudes? Developmental psychology, 39(1), 48.

Allport, G. W. (1979). The nature of prejudice. Reading, MA: Addison-Wesley.

Bar-Haim, Y., Ziv, T., Lamy, D., \& Hodes, R. M. (2006). Nature and nurture in own-race face processing. Psychological Science, 17(2), 159-163.

Baron, A. S. (2015). Constraints on the Development of Implicit Intergroup Attitudes. Child Development Perspectives, 9(1), 50-54.

Baron, A. S., \& Banaji, M. R. (2006). The development of implicit attitudes evidence of race evaluations from ages 6 and 10 and adulthood. Psychological Science, 17(1), 53-58.

Baron, A. S., \& Dunham, Y. (in press). Representing "Us" and "Them": Building blocks of intergroup cognition. Journal of Cognition and Development.

Bornstein, M. H. (1985). Habituation of attention as a measure of visual information processing in human infants: Summary, systematization, and synthesis. In G.Gottlieb \& N.A. Krasnegor (Eds.), Measurement of audition and vision in the first year of postnatal life: a methodological overview (pp. 253-300) Norwood, NJ: Ablex.

Bornstein, M. H., \& Sigman, M. D. (1986). Continuity in mental development from infancy. Child development, 251-274.

Brewer, M. B. (1979). In-group bias in the minimal intergroup situation: A cognitivemotivational analysis. Psychological Bulletin, 86(2), 307. 
Buttelmann, D., \& Böhm, R. (2014). The ontogeny of the motivation that underlies ingroup bias. Psychological Science, 25(4), 921-927.

Casstevens, R. (2007). jHab: Java habituation software (version 1.0. 2)[computer software]. Chevy Chase, $M D$.

Choi, J.-K., \& Bowles, S. (2007). The coevolution of parochial altruism and war. Science, $318(5850), 636-640$.

De Dreu, C. K., Greer, L. L., Van Kleef, G. A., Shalvi, S., \& Handgraaf, M. J. (2011). Oxytocin promotes human ethnocentrism. Proceedings of the National Academy of Sciences, 108(4), 1262-1266.

Devine, P. G. (1989). Stereotypes and prejudice: Their automatic and controlled components. Journal of Personality and Social Psychology, 56(1), 5.

Dovidio, J. F., Gaertner, S. E., Kawakami, K., \& Hodson, G. (2002). Why can't we just get along? Interpersonal biases and interracial distrust. Cultural Diversity and Ethnic Minority Psychology, 8(2), 88.

Drewnowski, A., Mennella, J. A., Johnson, S. L., \& Bellisle, F. (2012). Sweetness and food preference. The Journal of Nutrition, 142(6), 1142S-1148S.

Dunham, Y., Baron, A. S., \& Carey, S. (2011). Consequences of "minimal” group affiliations in children. Child Development, 82(3), 793-811.

Fazio, R. H., \& Olson, M. A. (2003). Implicit measures in social cognition research: Their meaning and use. Annual Review of Psychology, 54(1), 297-327.

Greenwald, A. G., McGhee, D. E., \& Schwartz, J. L. (1998). Measuring individual differences in implicit cognition: The implicit association test. Journal of Personality and Social Psychology, 74(6), 1464. 
Greenwald, A. G., Poehlman, T. A., Uhlmann, E. L., \& Banaji, M. R. (2009).

Understanding and using the Implicit Association Test: III. Meta-analysis of predictive validity. Journal of Personality and Social Psychology, 97(1), 17.

Halberda, J. (2006). Is this a dax which I see before me? Use of the logical argument disjunctive syllogism supports word-learning in children and adults. Cognitive Psychology, 53(4), 310-344.

Hamlin, J. K., \& Wynn, K. (2011). Young infants prefer prosocial to antisocial others. Cognitive Development, 26(1), 30-39.

Hamlin, J. K., Wynn, K., \& Bloom, P. (2007). Social evaluation by preverbal infants. Nature, 450(7169), 557-559.

Hamlin, J., Wynn, K., \& Bloom, P. (2010). Three-month-olds show a negativity bias in their social evaluations. Developmental Science, 13(6), 923-929.

Hamlin, J. K., Wynn, K., Bloom, P., \& Mahajan, N. (2011). How infants and toddlers react to antisocial others. Proceedings of the National Academy of Sciences, 108(50), 19931-19936.

Kagan, J. (1981). The second year: The emergence of self-awareness. Cambridge, MA: Harvard University Press.

Kelly, D. J., Quinn, P. C., Slater, A. M., Lee, K., Ge, L., \& Pascalis, O. (2007). The other-race effect develops during infancy evidence of perceptual narrowing. Psychological Science, 18(12), 1084-1089.

Kelly, D. J., Quinn, P. C., Slater, A. M., Lee, K., Gibson, A., Smith, M., et al. (2005). Three-month-olds, but not newborns, prefer own-race faces. Developmental Science, 8(6), F31-F36. 
Kinzler, K. D., Dupoux, E., \& Spelke, E. S. (2007). The native language of social cognition. Proceedings of the National Academy of Sciences, 104(30), 1257712580.

Kinzler, K. D., Shutts, K., DeJesus, J., \& Spelke, E. S. (2009). Accent trumps race in guiding children's social preferences. Social Cognition, 27(4), 623.

Kochanska, G., Casey, R. J., \& Fukumoto, A. (1995). Toddlers' sensitivity to standard violations. Child Development, 66(3), 643-656.

Kurzban, R., Tooby, J., \& Cosmides, L. (2001). Can race be erased? Coalitional computation and social categorization. Proceedings of the National Academy of Sciences, 98(26), 15387-15392.

Lai, C. K., Marini, M., Lehr, S. A., Cerruti, C., Shin, J. E. L., Joy-Gaba, J. A., ... \& Nosek, B. A. (2014). Reducing implicit racial preferences: I. A comparative investigation of 17 interventions. Journal of Experimental Psychology: General, 143(4), 1765.

LoBue, V., Rakison, D. H., \& DeLoache, J. S. (2010). Threat Perception Across the Life Span Evidence for Multiple Converging Pathways. Current Directions in Psychological Science, 19(6), 375-379.

McDonald, M. M., Navarrete, C. D., \& Van Vugt, M. (2012). Evolution and the psychology of intergroup conflict: the male warrior hypothesis. Philosophical Transactions of the Royal Society B: Biological Sciences, 367(1589), 670-679.

Patterson, M. M., \& Bigler, R. S. (2006). Preschool children's attention to environmental messages about groups: Social categorization and the origins of intergroup bias. Child Development, 77(4), 847-860. 
Pietraszewski, D., Cosmides, L., \& Tooby, J. (2014). The Content of Our Cooperation, Not the Color of Our Skin: An Alliance Detection System Regulates Categorization by Coalition and Race, but Not Sex. PloS One, 9(2), e88534.

Quinn, P. C., Yahr, J., Kuhn, A., Slater, A. M., \& Pascalis, O. (2002). Representation of the gender of human faces by infants: A preference for female. PerceptionLondon, 31(9), 1109-1122.

Raabe, T., \& Beelmann, A. (2011). Development of ethnic, racial, and national prejudice in childhood and adolescence: A multinational meta-analysis of age differences. Child Development, 82(6), 1715-1737.

Ramsey, J. L., Langlois, J. H., Hoss, R. A., Rubenstein, A. J., \& Griffin, A. M. (2004). Origins of a stereotype: Categorization of facial attractiveness by 6-month-old infants. Developmental Science, 7(2), 201-211.

Rand, D. G., Dreber, A., Ellingsen, T., Fudenberg, D., \& Nowak, M. A. (2009). Positive interactions promote public cooperation. Science, 325(5945), 1272-1275.

Rutland, A., Killen, M., \& Abrams, D. (2010). A new social-cognitive developmental perspective on prejudice the interplay between morality and group identity. Perspectives on Psychological Science, 5(3), 279-291.

Spelke, E. (1976). Infants' intermodal perception of events. Cognitive Psychology, 8(4), 553-560.

Tomasello, M., \& Vaish, A. (2013). Origins of human cooperation and morality. Annual Review of Psychology, 64, 231-255.

Vouloumanos, A., \& Werker, J. F. (2004). Tuned to the signal: The privileged status of speech for young infants. Developmental Science, 7(3), 270-276. 
Walker, A. S. (1982). Intermodal perception of expressive behaviors by human infants. Journal of Experimental Child Psychology, 33(3), 514-535.

Zajonc, R. B. (1968). Attitudinal effects of mere exposure. Journal of Personality and Social Psychology, 9(2, Part 2), 1-28.

Zajonc, R. B. (2001). Mere exposure: A gateway to the subliminal. Current Directions in Psychological Science, 10(6), 224-228. 